\title{
Stripe Correlations in $\mathrm{Na}_{0.75} \mathrm{CoO}_{2}$
}

\author{
J. Geck, ${ }^{1}$ M. v. Zimmermann, ${ }^{2}$ H. Berger, ${ }^{3}$ S. V. Borisenko, ${ }^{1}$ H. Eschrig, ${ }^{1}$ K. Koepernik, ${ }^{1}$ M. Knupfer, ${ }^{1}$ and B. Büchner ${ }^{1}$ \\ ${ }^{1}$ Leibniz Institute for Solid State and Materials Research IFW Dresden, Helmholtzstraße 20, 01069 Dresden, Germany \\ ${ }^{2}$ Hamburger Synchrotronstrahlungslabor HASYLAB at Deutsches Elektronen-Synchrotron DESY, \\ Notkestraße 85, 22603 Hamburg, Germany \\ ${ }^{3}$ Institut de Physique de la Matiére Complex (IPMC), EPF Lausannne, 1015 Lausanne, Switzerland
}

(Received 22 November 2005; published 7 September 2006)

\begin{abstract}
We present a combined high-energy x-ray diffraction and local-density approximation study of the sodium ordering in $\mathrm{Na}_{0.75} \mathrm{CoO}_{2}$. The obtained results rule out previously proposed Na-ordering models and provide strong evidence for the formation of sodium-density stripes in this material. The local-density approximation calculations prove that the sodium-density stripes lead to a sizable dip in the density of the Co states at the Fermi level, pointing to band structure effects as a driving force for the stripe formation. This indicates that the sodium ordering is connected to stripelike charge correlations within the $\mathrm{CoO}_{2}$ layers, leading to an astonishing similarity between the doped cuprates and the $\mathrm{Na}_{x} \mathrm{CoO}_{2}$ compounds.
\end{abstract}

DOI: 10.1103/PhysRevLett.97.106403

The many-body effects in correlated electron systems based on square lattices often result in the development of intrinsic charge inhomogeneities [1-3]. A particular prominent example is the so-called stripe ordered phase in the cuprates, where the doped holes condense into charge stripes within the physically relevant $\mathrm{CuO}_{2}$ planes [1]. Up to date, this stripe order is discussed intensively in relation to the high-temperature superconductivity and the anomalous metallic state of these materials.

Recently $\mathrm{Na}_{x} \mathrm{CoO}_{2}$, being a correlated electron system based on a triangular lattice, has attracted considerable attention due to the discovery of superconductivity in water-intercalated compounds [4] as well as the outstanding physics of the nonhydrated materials [5]. More specifically, for the nonhydrated $\mathrm{Na}_{x} \mathrm{CoO}_{2}$ materials with $x>0.5$ an anomalous metallic state with a thermopower 10 times larger than that of a typical metal, the coexistence of localized moments and electron itinerancy as well as unusual charge and spin order phenomena have been reported [6-8]. These extraordinary electronic properties cannot be reconciled with any conventional concept and remain to be a challenge for both theory and experiment.

This raises the question whether the unconventional physical properties of $\mathrm{Na}_{x} \mathrm{CoO}_{2}$ with $x \sim 0.7$ are also related to the presence of charge inhomogeneities, which are formed within the $\mathrm{CoO}_{2}$ layers in this case. To clarify this question, in the first step of this study, we performed a detailed characterization of structural modulations in $\mathrm{Na}_{0.75} \mathrm{CoO}_{2}$ by means of high-energy $\mathrm{x}$-ray diffraction (HXD). As has been proven by numerous previous experiments, HXD provides a highly sensitive probe for the extremely weak structural modulations related to the formation of charge inhomogeneities [9-11]. At the used photon energies around $100 \mathrm{keV}$, the $\mathrm{x}$-ray penetration depth is of the order of $1 \mathrm{~mm}$, which renders this technique insensitive to surface effects and guarantees the detection of bulk properties.
PACS numbers: 71.30.+h, 61.10.Eq, 64.60.Cn, 71.27.+a

For the present experiment, which was conducted at the beam line BW5 of the HASYLAB in Hamburg, the photon energy was set to $99.79 \mathrm{keV}(\lambda=0.124 \AA)$ and the longitudinal and transverse resolution at the (100) position was set to $0.009 \AA^{-1}$ (FWHM) and $0.015 \AA^{-1}$ (FWHM), respectively. Further details about the experimental setup can be found in the literature [12]. The high-quality $\mathrm{Na}_{0.75} \mathrm{CoO}_{2}$ single crystals used for the x-ray diffraction studies were grown by the sodium chloride flux methods as thoroughly described elsewhere [13]. The mosaicity spread of the sample was determined to be about $0.24^{\circ}$. Furthermore, the observed room temperature lattice parameters $a_{H}=2.83 \AA$ and $c_{H}=10.84 \AA$ of the hexagonal $P 6_{3} / m m c$ structure agree well with the values reported in the literature [14]. Since the $c$ axis depends strongly on $x$ [5], the resolution limited radial scan through the (004) reflection $\left(\mathrm{FWHM}=0.011^{\circ}\right)$ at $T=8 \mathrm{~K}$ verifies a welldefined and homogeneous sodium concentration in the probed sample volume.

During a survey in reciprocal space at $8 \mathrm{~K}$, a number of superstructure reflections were observed around the (100) reflection in the (HK0)-zone, which are summarized in Fig. 1. In this figure, the indicated reciprocal lattice vectors $\mathbf{b}_{1,2}$ correspond to the direct lattice vectors $\mathbf{a}_{1,2}$ that are parallel to the $\mathrm{Na}$ and $\mathrm{CoO}_{2}$ layers (cf. Fig. 4). Corresponding superstructures were also observed around the (110) position. Radial scans through the superstructure reflections at the commensurate $(1.5,-0.25,0)$ and $(2$, $-0.5,0)$ positions taken at $T=8 \mathrm{~K}$ are shown on the right-hand side of Fig. 1. The observation of superstructure reflections at equivalent positions together with the fact that these reflections do not vanish upon a rotation around the scattering vector, excludes multiple scattering as a possible origin for these peaks. The intensity profile at the $(1.5,-0.25,0)$ position was fitted by a single Lorentzian squared function. The FWHM determined by this fit together with the experimental resolution deter- 
(a)

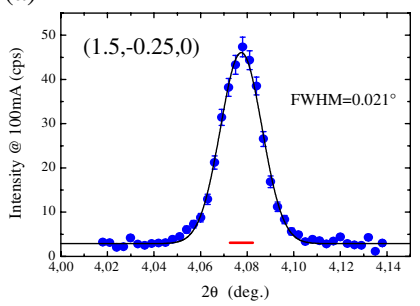

(b)

(c)

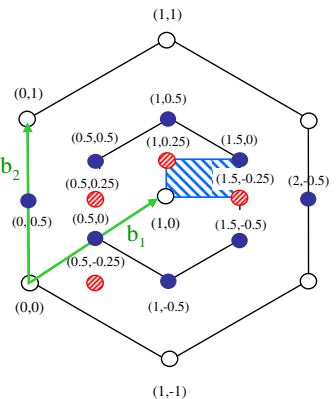

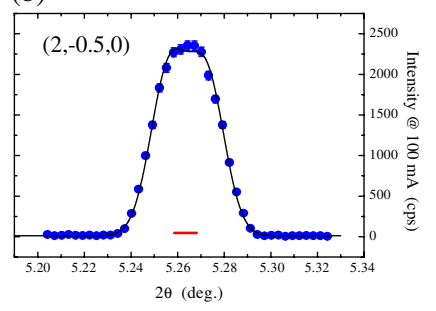

(d)

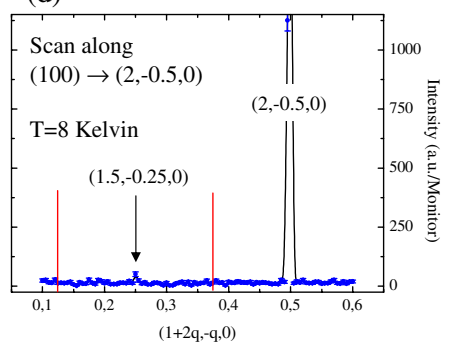

FIG. 1 (color online). (a), (b) Radial $(\Theta / 2 \Theta)$ scans taken at $T=8 \mathrm{~K}$. The small splitting of the $(2,-0.5,0)$ is due to the mosaic of the sample in combination with the finite experimental resolution. The solid lines are fits to the data (see text) and the horizontal bar represents the experimental resolution. (c) Summary of observed superstructure peaks around (100). The hatched rectangle marks the assumed unit cell of the reciprocal lattice. Open, solid, and hatched circles indicate Bragg reflections, superstructure peaks observed for all investigated temperatures, and peaks only observable below $T_{\text {So }}$, respectively. (d) Line scan along the $(100) \rightarrow(2,-0.5,0)$ direction. Vertical lines: positions where peaks are expected, according to electron diffraction.

mined at the (100) position yield a correlation length of about $300 \AA$ for the corresponding superstructure modulation.

In order to obtain information about the origin of the observed superstructures, we performed temperature dependent measurements at two representative positions in reciprocal space: in Fig. 2, the temperature dependence of the integrated intensity at the $(1.5,-0.25,0)$ position is displayed, showing that this reflection appears only below $T_{\mathrm{SO}}=350 \mathrm{~K}$. In addition to this, the intensity of the (1.5, $-0.25,0)$ reflection depends strongly on the cooling process, as demonstrated by the two measurements shown in Fig. 2. The first measurement was performed with increasing temperature after the sample had been cooled down slowly at $2 \mathrm{~K} / \mathrm{min}$ (open symbols). The second measurement was also taken upon heating, but this time the sample had been cooled down rapidly at about $15 \mathrm{~K} / \mathrm{min}$ (closed symbols). The large differences between the two measurements are evident. This is a strong indication for an ordering phenomenon that is related to the ordering of sodium ions, where relatively slow ion-hopping processes are involved. Upon rapid cooling, the sodium order is frozen, i.e., a certain amount of disorder remains, whereas it can be established to a higher extent if the sample is cooled down slowly. Upon increasing the temperature again, the ther-

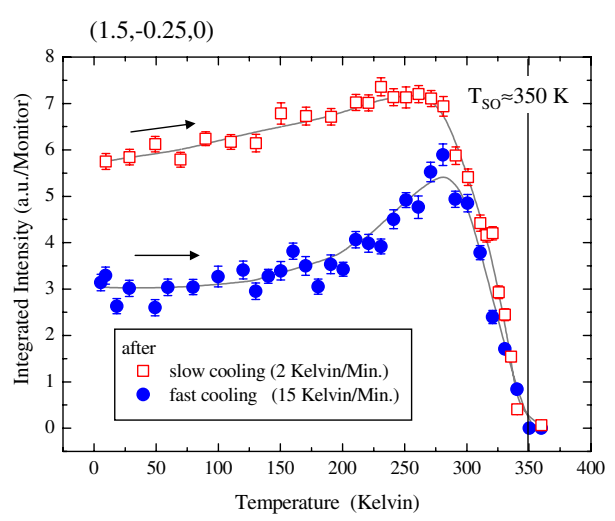

FIG. 2 (color online). Temperature dependence of the integrated intensity at the $(1.5,-0.25,0)$ position. After cooling down to low temperatures at different cooling rates, both measurements have been performed at increasing temperature. The solid lines are guides to the eye.

mally activated sodium ions become more and more mobile, which allows to remove defects in the sodium superlattice before the order finally vanishes upon heating at $T_{\text {SO }}$. The conclusion that the superlattice modulation below $T_{\mathrm{SO}}$ is not primarily related to charge order within the $\mathrm{CoO}_{2}$ layers but to sodium order is further supported by the metallic resistivity below $350 \mathrm{~K}$ of $\mathrm{Na}_{x} \mathrm{CoO}_{2}$ around $x=0.75$ [5] and a recent neutron scattering study [14], where changes in the $\mathrm{Na}$ sublattice were observed in the very same temperature regime. In addition, $T_{\mathrm{SO}}$ matches the temperature of the first order transition found in a recent thermodynamic study on $\mathrm{Na}_{0.75} \mathrm{CoO}_{2}$ [15].

As a second reflection, we have studied the $(2,-0.5,0)$ peak and its temperature dependence. The integrated intensity of this reflection measured after rapid cooling is shown in Fig. 3. The measurement after rapid cooling shown in Fig. 2 and the data given in Fig. 3 have been obtained during the same run, verifying a completely different temperature dependence of the two reflections.

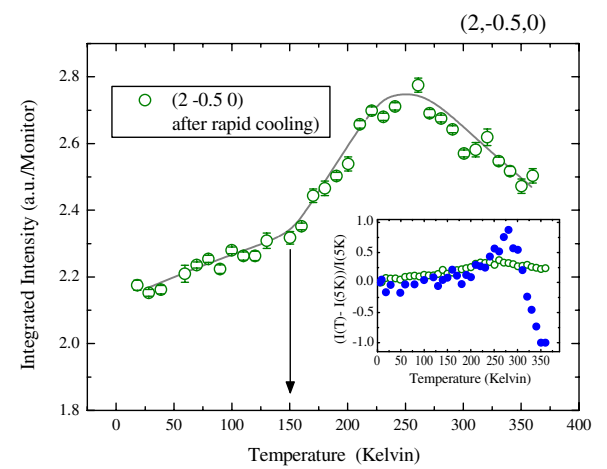

FIG. 3 (color online). Temperature dependence of the integrated intensity at the $(2,-0.5,0)$ position $(\bigcirc)$. The measurement has been performed with increasing temperature after rapid cooling. The solid line is just a guide to the eye. A comparison of the relative intensity changes for the $(1.5,-0.25,0)(\mathbf{O})$ and the $(2,-0.5,0)$ reflection is given in the inset. 
As can be observed in Fig. 3, the $(2,-0.5,0)$ reflection does not disappear above $350 \mathrm{~K}$, but is observed in the whole investigated temperature range instead. The slope change of the $(2,-0.5,0)$ intensity around $150 \mathrm{~K}$ roughly coincides with the temperature regime where the (1.5, $-0.25,0)$ intensity starts to increase considerably. Furthermore, the intensity at the $(2,-0.5,0)$ reflection also varies across the phase transition at $350 \mathrm{~K}$, indicating that these two superlattice modulations are coupled. Nonetheless, the temperature dependent variation of the $(2,-0.5,0)$ reflection is relatively weak, as illustrated in the inset of Fig. 3. Also the width of this reflection remains constant within the errors of the experiment. To conclude so far, the low temperature superstructure is established in two steps: first, the doubling of the unit cell signaled by the $(2,-0.5,0)$ reflection occurs at temperatures well above $360 \mathrm{~K}$ and then, the structural modulation related to the $(1.5,-0.25,0)$ peak appears below $T_{\mathrm{SO}}=350 \mathrm{~K}$, which can clearly be attributed to sodium order.

As indicated in Fig. 1, the observed superlattice reflections in the (HK0) zone can be described by the orthogonal basis vectors $\mathbf{b}_{1} / 2-\mathbf{b}_{2} / 4$ and $\mathbf{b}_{2} / 4$. But since only a finite number of positions in reciprocal space could be checked and, furthermore, differently oriented twin domains might exist in the sodium ordered phase, the HXD data do not allow to prove unambiguously the dimensions of the unit cell.

Nonetheless, the HXD data yield important clear-cut conclusions regarding previously proposed models for the sodium ordering in $\mathrm{Na}_{0.75} \mathrm{CoO}_{2}$ : the observed superlattice reflections do not correspond to the unit cell which was deduced from electron diffraction (ED) data [16] and which was used later for $a b$ initio calculations $[17,18]$. In particular, the $(100) \rightarrow(2,-0.5,0)$ line scan in Fig. 1(d) verifies the absence of superlattice reflections corresponding to this unit cell. The superstructure related to the vacancy ordering that has been found in Monte Carlo simulations [19] does also not match the HXD results.

Regarding the complications related to the twinning of the samples, we note that the different temperature dependencies in Fig. 2 might be related to a twin structure that depends on the cooling process. These effects are currently under study and will be the subject of a forthcoming publication. However, it can be observed in Fig. 1 that the (110) direction differs from the $(100) /(010)$ direction. Since this would not be the case in a fully twinned sample [see, e.g., Ref. [18] ], the data indicate that a single twin domain dominates the HXD intensity.

Therefore, we performed a local-density approximation (LDA) study of the sodium order based on the supercell of the direct lattice shown in Figs. 4(a) and 4(b), which corresponds to reciprocal lattice vectors $\mathbf{b}_{1} / 2-\mathbf{b}_{2} / 4$ and $\mathbf{b}_{2} / 4$. The aim of these calculations was threefold: (i) to determine the lowest energy state in this supercell, (ii) to compare the obtained total energies to the proposed models in the literature, and (iii) to determine the effect of the sodium order on the electronic states of the $\mathrm{CoO}_{2}$ planes.

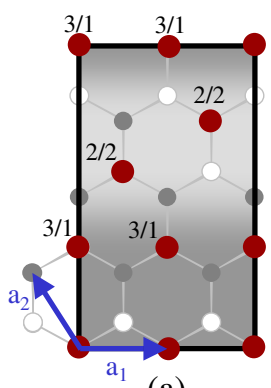

(a)

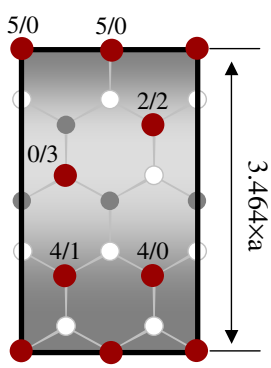

(b)

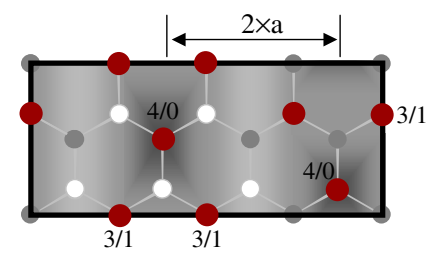

(c)

FIG. 4 (color online). Models for the sodium superlattice in $\mathrm{Na}_{0.75} \mathrm{CoO}_{2}$. Projections of superstructure unit cells on the $\left(\mathbf{a}_{1}, \mathbf{a}_{2}\right)$ plane are shown. Small gray and white circles indicate $\mathrm{Na}(1)$ and $\mathrm{Na}(2)$ sites, respectively. The occupied $\mathrm{Na}$ sites are indicated by big solid (red) circles. The numbers $m / n$ indicate the number of first or second $\mathrm{Na}-\mathrm{Na}$ neighbors and the corresponding sodium-density variations are indicated by the gray shadings.

For the LDA [20] study the FPLO package version 5.20 [21] has been used. The calculations were performed non-spinpolarized and all calculations were done for a fixed unit cell volume. To compare to previous calculations in the literature [18], we used $a_{H}=2.82 \AA$ and $c_{H}=10.89 \AA$, which slightly differ from the observed values by about $0.5 \%$.

The free parameter of the oxygen Wyckoff position was relaxed for each structure and resulted in the same oxygen position for all $\mathrm{Na}$ patterns considered. In order to assess our calculations we recalculated the total energies of a number of structures considered in Ref. [17] and obtained very good agreement in the structural energy differences. The LDA calculations yield the lowest total energy for the structure given in Fig. 4(a), which amounts to $E_{a}=$ $45.2278 \mathrm{eV}$ per $\mathrm{Na}_{0.75} \mathrm{CoO}_{2}$ formula unit. The two structures shown in Fig. 4(b) and 4(c) correspond to higher total energies. We obtain $E_{b}-E_{a}=35.4 \mathrm{meV}$ and $E_{c}-E_{a}=$ $46.3 \mathrm{meV}$, respectively. With respect to the stacking in the third direction, two possibilities were considered with an inversion center at the Co site and midway between two nearest neighbor Co sites, respectively. However, the corresponding energy differences are negligible. The differences between the structures (a), (b) and (c) can essentially be understood by Coulomb energy arguments: (a) and (c) comprise a charge density wave of comparable amplitude, but with wave vector $2 \mathbf{a}_{1}+4 \mathbf{a}_{2}$ in the case (a), while (c) has the shorter wave vector $2 \mathbf{a}_{1}$. The structure (b) has also a charge density wave with wave vector $2 \mathbf{a}_{1}+4 \mathbf{a}_{2}$, however with a considerably larger amplitude. 
The model reproduced in Fig. 4(c) corresponds to the lowest energy state reported by Zhang et al. based on the unit cell deduced from ED data [16,17]. Since this model neither corresponds to the superstructure reflections observed by HXD nor has the lowest total energy, we can exclude this ordering pattern as a ground state for $\mathrm{Na}_{0.75} \mathrm{CoO}_{2}$. Exactly the same holds for the structure determined by Meng et al. [18]; i.e., it does not agree with the HXD results and we find a higher total energy in LDA, comparable to the one of the ordering in Fig. 4(c). Hence, this ordering is ruled out as well.

Next, we compare our results to existing experimental data: the disagreement between the ED and the HXD data mentioned above could be due to the different sample volumes probed by the two techniques (the penetration depth of $100 \mathrm{keV}$ photons is of the order of $1 \mathrm{~mm}$, while it is of the order of $100 \mathrm{~nm}$ in the case of ED). Another complication might be related to the fact that the electron beam used for the ED experiment itself can influence the sodium ordering [16].

Concerning existing neutron data of $\mathrm{Na}_{0.75} \mathrm{CoO}_{2}$, the transition temperature $T_{\mathrm{SO}}$ found in the present HXD study is in good agreement with the sodium rearrangement that was observed by neutron powder diffraction, as mentioned above [14]. However, in this study only a symmetry change related to the $\mathrm{Na}$ sites was revealed, whereas no enlargement of the unit cell was reported. The $\mathrm{Na}(2) / \mathrm{Na}(1)$ ratio of the ordering depicted in Fig. 4(a) (and also of the model by Zandbergen et al.) is $\gamma=1$, whereas a value of $\gamma=2.5$ was obtained by a refinement of the neutron data. A possible origin for the different $\gamma$ values might lie in deviations from the ideal ordering pattern which become increasingly important around $x=0.75$ [17]. Indeed, the sodium disorder was found to be strongly increased in $\mathrm{Na}_{0.75} \mathrm{CoO}_{2}$ polycrystals as compared to single crystalline materials [15]. This provides an explanation for the different $\gamma$ values and is consistent with the fact that no superlattice has been found in the neutron powder diffraction studies.

The obtained lowest energy state (a) exhibits sodiumdensity stripes (SDS) within the Na planes, as indicated by the gray shading in Fig. 4(a). The LDA calculations prove that this ordering produces a sizeable dip in the density of the Co states at the Fermi level, pointing to band structure effects as a driving force for the stripe formation. This indicates that the SDS are related to intrinsic stripelike charge correlations within the $\mathrm{CoO}_{2}$ planes, which is supported by a NMR/NQR study, where different Co sites were observed for $\mathrm{Na}_{x} \mathrm{CoO}_{2}$ with $x \approx 0.7$ [22]. The coupling of the SDS to the $\mathrm{CoO}_{2}$ planes is also corroborated by the development of a SDW in $\mathrm{Na}_{0.75} \mathrm{CoO}_{2}$ below $22 \mathrm{~K}$ [15].

Taking into account that the hydrated superconducting samples have been shown to be of the composition
$\mathrm{Na}_{0.337}\left(\mathrm{H}_{3} \mathrm{O}\right)_{z} \mathrm{CoO}_{2} \cdot y \mathrm{H}_{2} \mathrm{O}$ with a doping level corresponding to $\mathrm{Na}_{x} \mathrm{CoO}_{2}$ with $x \approx 0.7$ [23], an astonishing analogy to the high-temperature superconducting cuprates appears: the electric field that is caused by the SDS might induce a pinning potential for charge stripes within the adjacent $\mathrm{CoO}_{2}$ layers, similar to the cooperative octahedral tilts in the so-called LTT phase of the doped cuprates. One may speculate whether the reduction of this pinning potential upon hydration is related to the concomitant appearance of superconductivity. However, this scenario has certainly to be verified by further experiments which are capable to detect directly charge order within the $\mathrm{CoO}_{2}$ layers.

In conclusion, combining experimental HXD data and LDA model calculations we can clearly rule out previous proposed sodium order models for $\mathrm{Na}_{0.75} \mathrm{CoO}_{2}$. Furthermore, the experimental and theoretical results provide firm evidence for the development of SDS below $T_{\mathrm{SO}}$, which is related to charge-stripe correlations within the twodimensional triangular $\mathrm{CoO}_{2}$ layers.

We would like to thank Dr. Bussy (University of Lausanne) for the microprobe analysis. This work was supported by the Swiss NCCR research pool MaNEP of the Swiss NSF and the DFG with the SFB 463, FOR 538, and DFG KL1824/2 research projects.

[1] J. Orenstein and A. Millis, Science 288, 468 (2000).

[2] Y. Tokura and N. Nagaosa, Science 288, 462 (2000).

[3] Y. Tokura, Phys. Today, 56, No. 7, 50 (2003).

[4] K. Takada et al., Nature (London) 422, 53 (2003).

[5] M. L. Foo et al., Phys. Rev. Lett. 92, 247001 (2004).

[6] Y. Wang et al., Nature (London) 423, 425 (2003).

[7] J. Gavilano et al., Phys. Rev. B 69, 100404(R) (2004).

[8] C. Bernhard et al., Phys. Rev. Lett. 93, 167003 (2004).

[9] T. Niemoeller et al., Eur. Phys. J. B 8, 5 (1999).

[10] M. v. Zimmermann et al., Europhys. Lett. 41, 629 (1998).

[11] A. Vigliante et al., Phys. Rev. B 56, 8248 (1997).

[12] R. Bouchard et al., Synchrotron Radiation News 5, 90 (1998).

[13] M. N. Iliev et al., Physica (Amsterdam) 402C, 239 (2004).

[14] Q. Huang et al., Phys. Rev. B 70, 134115 (2004).

[15] B. Sales et al., Phys. Rev. B 70, 174419 (2004).

[16] H. W. Zandbergen et al., Phys. Rev. B 70, 024101 (2004).

[17] P. Zhang et al., Phys. Rev. B 71, 153102 (2005).

[18] Y. Meng et al., Phys. Rev. B 72, 172103 (2005).

[19] M. Roger et al., cond-mat/0507040.

[20] J. P. Perdew and Y. Wang, Phys. Rev. B 45, 13244 (1992).

[21] K. Koepernik and H. Eschrig, Phys. Rev. B 59, 1743 (1999).

[22] I. R. Mukhamedshin et al., Phys. Rev. Lett. 94, 247602 (2005).

[23] C. J. Milne et al., Phys. Rev. Lett. 93, 247007 (2004) 